\title{
Development Of A Reduced Order Model For Standard-Based Measurement And Verification To Support ECM
}

\author{
Alessandro Piccinini ${ }^{1,2}$, Letizia D'Angelo ${ }^{1,2}$, Federico Seri ${ }^{1,2}$, Conor Deane ${ }^{1,2}$, Raymond Sterling ${ }^{1,2,4}$, \\ Andrea Costa ${ }^{3}$, Alberto Giretti ${ }^{5}$, Marcus M. Keane ${ }^{1,2}$ \\ ${ }^{1}$ Informatics Research Unit for Sustainable Engineering (IRUSE), NUI Galway, Ireland \\ ${ }^{2}$ Department of Civil Engineering, NUI Galway, Ireland \\ ${ }^{3}$ R2M Solution Srl, Pavia, Italy \\ ${ }^{4}$ R2M Solution SL, Madrid, Spain \\ ${ }^{5}$ Department of Civil and Building Engineering and Architecture, UNIVPM, Ancona, Italy
}

\begin{abstract}
This paper describes a methodology which utilises a Reduced Order Model (ROM) in overcoming typical barriers related to the Energy Performance Contracting (EPC) such as lack of building information and uncertainty regarding post renovation. For this purpose, a ROM supported by a JModelica.org Python script (ROMFit) for the optimization and calibration process has been utilised in the Measurement and verification $(\mathrm{M} \& \mathrm{~V})$ process. The accuracy and benefits of this model are demonstrated by a comparison with an IES-VE Whole Building Energy Simulation Model. The results presented in this paper show that the ROM proves to be the most accurate model with regards to energy demand forecasting and furthermore, it is useful in estimating numerous energy savings of retrofitting scenarios.
\end{abstract}

\section{Introduction}

Within the last decade, the European Union (EU) has developed policies aimed at accelerating the costeffective renovation of existing buildings, with the vision of a decarbonised building stock by 2050 (European Union Directive, 2018).

One of the potential measures to target this objective and enhance the energy efficiency (EE) of buildings is Energy Performance Contracting (EPC) (Augustins et al., 2018). However, there are different risks and barriers opposing the uptake of EPC such as process complexity, lack of information, uncertainty about post-renovation energy performance, access to finance, lack of trust in Energy Service Companies (ESCO), and lack of skilled professional, fragmentation of value chain and unclear financial mechanisms.(Lee et al., 2015)

One way to tackle the barriers posed by the aforementioned uncertainties is the utilisation of Measurement and Verification $(\mathrm{M} \& \mathrm{~V})$ protocol. $\mathrm{M} \& \mathrm{~V}$ is a procedure which measures and analyses data needed to verify and report energy savings within a system or a whole facility. $M \& V$ underpins and enhances a standardsbased approach to the implementation of energy conservation measures (ECM's). Guidelines regarding the $\mathrm{M} \& \mathrm{~V}$ protocol are provided by the International Performance Measurement and Verification Protocol (IPMVP ${ }^{\circledR}$ ) (Efficiency Valuation Organization, 2016). This protocol defines a standards-based approach to estimate the potential and actual savings and can be used to quantify the payments to all stakeholders throughout the EPC process. In verifying the results of energy efficiency, water efficiency, and renewable energy projects, the IPMVP ${ }^{\circledR}$ provides four options referenced from A-D. An overview of these methods and practices is proposed by Ginestet and Marchio (2010). One of the main recommendations of the IPMVP ${ }^{\circledR}$ guidelines is that the $\mathrm{M} \& \mathrm{~V}$ costs does not exceed $10 \%$ of the average annual savings achieved through its application. Additional cost limits are provided by $\mathrm{M} \& \mathrm{~V}$ guidelines, such as the M\&V handbook (Air Force Civil Engineer Center ESPC Playbook, 2017), where the cost limits range from a minimum of $1 \%$ of the annual measured savings for the IPMVP ${ }^{\circledR}$ Option A to a maximum of $10 \%$ for the IPMVP ${ }^{\circledR}$ Option D.

Option D is the development of a calibrated computer simulation model that supports detailed analysis of various ECM's. According to IPMVP ${ }^{\circledR}$, this procedure should be undertaken for three main reasons: 1) for projects that generate enough savings to justify its use; 2) when creating a baseline is not possible for other options (e.g. new constructions, or major building renovation): 3 ) when the ECM's responsible for the savings are not easy to measure. This option also provides a big opportunity to evaluate the savings of each ECM or multiple ECMs and thus, to test the best renovation scenario to apply to a building.

Many software tools are suggested in the IPMVP $^{\circledR}$ to analyse energy consumption and determine building control and operation opportunities., these range from eQUEST (2018), EnergyPlus (2018), or Integrated Environmental Solutions (2018) (IES-VE). Software tools such as these are classified as Whole Building Energy Simulation Modelling (WBESM) or White Box Models. These modelling approaches can provide the most comprehensive prediction of building energy performance with a vast range of detailed outputs from energy consumption to indoor comfort. These models are also suitable for retrofit analysis as the detailed physicsbased equations can be used to model and implement building components, systems and the subsystems prior to any renovation. However, as demonstrated in several papers, the high computational time ( $\mathrm{Li}$ and Wen, 2014), the complexity, the cost of the model implementation, and the uncertainty of the model parameters (Foucquier $e t$ al., 2013) means this procedure is not a popular method adopted by IPMVP ${ }^{\circledR}$ and in general for thermal prediction of existing building in the deep-renovation processes (Harb et al., 2016). All issues aforementioned also carry 
the increasing cost burdens which of this option that usually exceed cost limit recommendations.

Two alternatives from this purely physics modelling approach (white-box) can be found in 1) A purely empirical approach (black-box) and 2) A synthesis of White and Black box modelling approaches into a greybox modelling approach (Coakley et al., 2014).

The black box approach is data driven and does not require knowledge about the system. It includes monitoring the building and implementing a simple mathematical or statistic model (e.g. ANN, ARX, etc). For this approach, accuracy is achieved through the requirement of a large amount of high-quality data. Although these models are based on trained data, the model variables are abstract and do not have a physical meaning (Reynders et al., 2015) since the weight of those variables depends on the sensibility on the output variables. In addition to this, building parameters are not the same for each building which means this approach requires unique building parameters for each model. Since this model is based on measured data, the approach has limited use in new constructions in addition to major building modifications. Moreover, it is difficult to physically interpret building model. Thus, their ability to predict building behaviour after thermal improvements or altering control strategies cannot be demonstrated (Berthou et al., 2014) .

The final approach is provided by grey box or hybrid models. These models will be explained in detail in the Section 2 and are considered as a hybrid where physical equations and physical knowledge about the system is used to define the model structure and the unknown parameters are estimated using statistical methods.

This research paper attempts to overcome the EPC barriers discussed so far by offering a novel methodology to support the IPMVP ${ }^{\circledR}$ by developing and utilising a Python script (ROMFit) based on Grey box model or Reduced Order Model (ROM) that supports accurate and reliable quantification of energy savings achieved through ECMs. As described in the next section, a grey box model was selected for the capability of these models of estimating the energy consumption in the typical EPC scenario where the technical information about the building are missing and a high uncertainty of the model parameters is present (Giretti et al. 2018). Meanwhile ROMFit was developed to help accomplish IPMVP $^{\circledR}$ requirements by improving the efficiency of the calibration procedure. To demonstrate the accuracy of the methodology, the ROM proposed has been compared with a whole facility linear regression (Option $C$ ) and a white box model developed with IES-VE (Option D).

In Section 2, an overview of grey box modelling approaches is presented. Section 3 presents the model used in the analysis. Section 4 outlines the methodology used to accomplish the IPMVP ${ }^{\circledR}$ protocol. Section 5 provides a detailed description on the pilot case study. Simulations, results and comparisons with standard IPMVP $^{\circledR}$ methods are shown in Section 6 and finally the Section 7 describes conclusions and future works.

\section{Grey Box Models}

Grey box models are a synthesis of white and black box models. They consist of coupling of the physical meaning and structure from the white box paradigm and the statistical approach and parameter estimation from the black box approach thus tacking the advantages of both methods. In general, the grey box model structure simplifies the physical description of the building using thermal network analogies and treating the system complexity as an electrical problem by means of resistances and capacitances where the thermal mass of the building is lumped to a discrete number of capacitances based on the model type (Reynders et al., 2014). The number of capacitances, not counting for the air mass, give the order of the model as extensively explained in ( M. Lauster et al., 2014) .

All the characteristics described so far, highlights the top features of the grey box method, having both physical meaning and a high grade of generality. In addition, these characteristics provide the capability of having parameters related to building component and the opportunity to use the same model structure in different kinds of buildings. Most recently, this kind of model has become increasingly popular in evaluating the environmental and energy performance of buildings. One of the most compressive comparison of grey box models accuracy was provided by Bacher and Madsen (2011), similar studies have been done by Fux et al., (2014), ,Reynders et al. (2014), Berthou et al. (2014). Nonetheless, there are still numerous problems with the use of this model approach.

The first problem is associated with the estimation of the uncertain parameters. This uncertainty usually derives from errors and approximations in data measurement and parameter estimation, material degradation, lack of knowledge of the building. In the papers cited above, this uncertainty is fixed by applying algorithms such as the maximum likelihood estimation method and the Kalman filter. However, all these methods do not give any physical meaning to the calibrated parameters, making it difficult to understand and explain the whole calibration process.

The second problem is due to historical data used for the parameters estimation which is usually taken in to account without using knowledge about the building. These issues make quite difficult in using the model in the renovation phase where it is important to have a full physical meaning of each parameters in order to modify them for when developing renovation packages.

A proposed solution to these gaps has been outlined by Lauster at al. in (M. Lauster et al., 2014), using Modelica to compare the first order grey box model of the EN ISO 13790 to the second order grey box model of the Guideline VDI 6007. The second model has been used by the same authors as a baseline to create the different Low Order Models of the open source Modelica Library AixLib (Müller et al., 2016). From this library a tool called TEASER (Remmen et al., 2018) has been created. This tool is used to generate and simulate Modelica 
models at both building and district level. It also allows the application of retrofit scenarios. However, it is unclear at this stage as to how the TEASER Tool might be incorporated into a formal EPC business process model and in the application of the $\mathrm{M} \& \mathrm{~V}$ protocol.

Due to this concern, an extension of the third order building model proposed in Giretti et al. (2018) is assumed as it was successfully used for a EPC tendering phase. The extended model used to meet the IPMVP ${ }^{\circledR}$ protocol purpose is described in the next section.

\section{Model Description}

The model proposed in this paper is developed using the Modelica language. The selection of this language was due to three main reasons. Firstly, it allows one to model complex dynamic energy systems supported by an objectoriented modelling and simulation. Secondly, the language recognizes linear, non-linear and hybrid equations and finally, many open source and commercial simulation environments support Modelica providing several numerical solvers, algorithms and libraries such as the IBPSA Project 1 Library (2018), which has been implemented in the model.

The ROM is composed of the following four main components: 1) Internal Gains 2) Heating and Cooling. 3) Building and 4) Weather.

A Tool has been created to estimate the parameters needed for the model. This Tool uses information obtained during the tendering phase through data collection and on-site site surveys. In case of missing data, standardized packages and associated parameter values have been provided to support good model parameters estimation.

Since the model considers the main physical dependencies among each variable, the calibration phase is far less complicated than the white-box approach. A dedicated Python script (ROMFit) has been developed to simplify the parameter insertion and increase the speed and accuracy of the calibration procedure utilising an FMU file (Functional Mock-up Interface,2018) generated from JModelica.org (2018).

\section{Internal Gain Component}

The Internal Gain component is modelled to evaluate the thermal heat power of people, lighting and equipment in the building. The inputs needed to estimate these parameters are the occupancy schedule, the maximum load power of the three internal heat gains and the standby consumption of the equipment. Furthermore, a Modelica component capable of estimating the electrical consumption of lighting and miscellaneous equipment has been included and is used for the estimation of the total energy consumption.

\section{Heating and Cooling Component}

The Heating and Cooling component is implemented as a thermostatically regulated heat gain with an internal control loop. In expanding the use of the model to residential dwellings, an input related to the occupancy schedule has been added to the model proposed in Giretti et al. (2018). Additionally, two statistical parameters have been included to maximize the efficiency of the auto-calibration engine.

\section{Building Component}

The main element of the ROM is the Building component. The structure of this component is derived from Giretti's model (Giretti et al., 2018) which is an extension of one of the model proposed by Madsen (Bacher and Madsen, 2011).

The model, as shown in Figure 1, is created using the Modelica language and is composed of 14 resistances, 3 capacitances, a solar irradiation component, a natural ventilation component and an air infiltration component. The aforementioned elements are connected to a Modelica MixingVolume element that represents the entire volume of the building. This element is also connected to the outdoor Weather Data and thus receives inputs such as temperature and solar irradiation through the weather data component. Finally, the MixingVolume component is connected to the Internal Gain Component and the Heating and Cooling Component through the two heat ports.

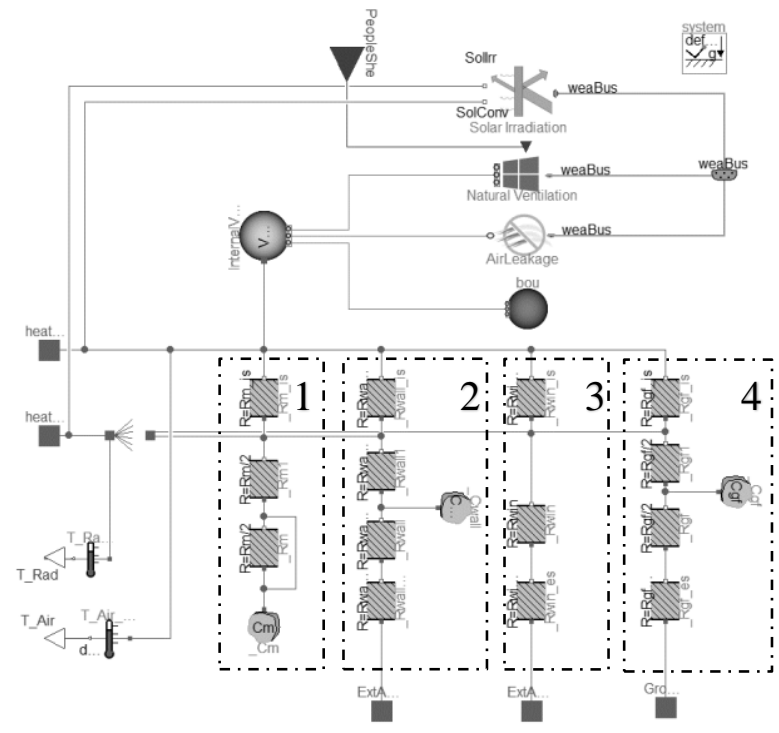

Figure 1-Modelica ROM, Building Component

The resistances and capacitances in this element can be divided in four groups and they are used to lump all the building internal and external envelope. The first assembly ( $\mathrm{Cm}, \mathrm{Rm}, \mathrm{Rm} 1, \mathrm{Rm} \_$is) is used to lump the building internal wall and slabs. The second group (Rwall_es, Rwall, Cwall, Rwall1, Rwall_is) is used to lump the whole building opaque envelope. The third (Rwin_es, Rwin, Rwin_is) represents the building transparent envelope and finally the forth lump (Rgf_es, Rgf, Cgf, Rgf1, Rgf_is) is the building ground floor. These elements take the temperature inputs from the Weather Data element.

The solar irradiation component uses two elements of the IBPSA Project 1 Library (2018) to compute the direct solar irradiation and the diffuse solar irradiation using the Perez's anisotropic sky model (Perez et al., 1990). The irradiation is then reduced, by using the $G$ total value calculated according to the BS EN 13363-1 (2003). 
The natural ventilation component has been modelled using a formula from the Ashrae Handbook Fundamentals (2009) which is based on temperature and pressure difference between the ambient and the internal volume.

Finally, to compute the air infiltration in the building an air leakage component has been created using as basis component of the IBPSA Project 1 Library (2018).

\section{Methodology}

An overview of the model usage process is shown Figure 2 , the procedure is composed by three main steps:

1. The Tool for the ROM parameters estimation which receives building data as input;

2. A .fmu file of the Modelica model described in the section above;

3. The ROMFit Python script for the model simulation and the automatic calibration.

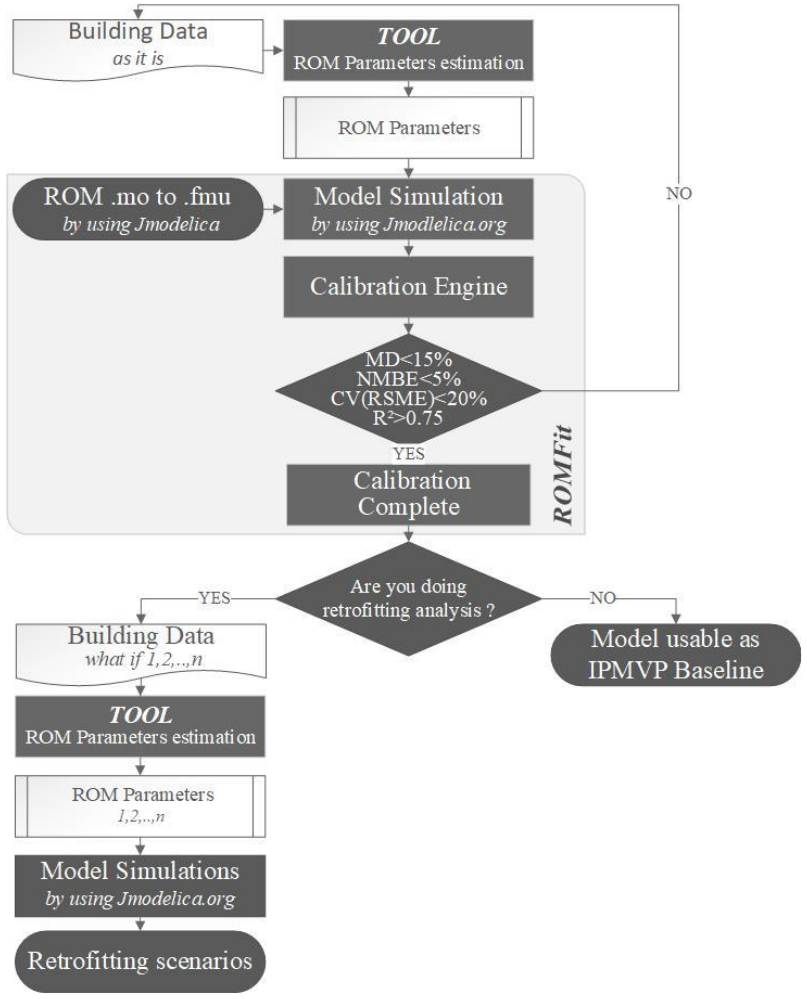

Figure 2 - ROM methodology flow chart

The Tool used to estimate the parameters, discussed in the previous section, is based on widely used method which is defined in standards. For instance, the resistances of both the internal and the external walls and facades are calculated as specified in ISO 6946 (2007), the internal and external windows heat loses are calculated using the $\mathrm{U}$-value of the windows, and by using a simplified version of the ISO 10077 (2017). The resistance values of the ground floor are calculated with a simplified version of the ISO 13370 (2017), thus the G-total value is calculated from standard configuration by using the BS EN 13363-1 (2003).The thermal resistances and capacitances are calculated from standard values, materials properties and construction composition. For the profiles generation, a graphical interface has been included in the Tool and the numerical output needed by the model is given.
The ROMFit script is created using Python and Jmodelica, a code is developed to simplify the insertion of the parameters including the model simulation and calibration. Jmodelica, which is the platform used to build the code, relies on Functional Mock-up Interface (FMI) for simulation purposes. Thus, using this platform an .fmu file has been created from the reduced order model .mo file. The .fmu file generated has been uploaded in the ROMFit script where the parameters calculated with the Tool previously discussed can be quickly and simply inserted. By using ROMFit the model is simulated and then, after the first simulation, an engine is to adjust the uncertain parameters and simplifies the model calibration and its verification with energy consumption data.

The uncertain parameters are selected following the assumption made in Giretti et al. (2018) paper. Since there are a restricted number of these parameters (Table 4), the calibration engine makes an iterative procedure that consists of changing the uncertain parameters within a range of values with and a prechosen step. For each of the parameter selection, a simulation is run and then the resulting baseline model energy consumption simulated is automatically compared with the actual energy consumption data, and, as suggested by IPMVP $^{\circledR}$ the following statistical values are verified:

- $\mathrm{NMBE}<5 \%$

$$
N M B E=\frac{1}{\bar{Y}} \sqrt{\frac{\sum_{i=1}^{n}\left(\hat{Y}_{i}-Y_{i}\right)}{n-1}} * 100(\%)
$$

- $\operatorname{CV}(\mathrm{RMSE})<20 \%$

$$
\begin{gathered}
C V(R M S E)=\frac{1}{\bar{Y}} \sqrt{\frac{\sum_{i=1}^{n}\left(\hat{Y}_{i}-Y_{i}\right)^{2}}{n-1}} * 100(\%) \\
R^{2}=\left(\frac{n \cdot \sum_{i=1}^{n} \hat{Y}_{i} \cdot Y_{i}-\sum_{i=1}^{n} \hat{Y}_{i} \cdot \sum_{i=1}^{n} Y_{i}}{\sqrt{\left(n \cdot \sum_{i=1}^{n} \hat{Y}_{i}^{2}-\left(\sum_{i=1}^{n} \hat{Y}_{i}\right)^{2}\right)\left(n \cdot \sum_{i=1}^{n} Y_{i}^{2}-\left(\sum_{i=1}^{n} Y_{i}\right)^{2}\right)}}\right)^{2} \\
\text { - monthly deviation }<15 \% \\
M D=\frac{\left(\hat{Y}_{i}-Y_{i}\right)}{Y_{i}} * 100(\%)
\end{gathered}
$$

In these equation $\hat{Y}$ is the simulated value of the actual value $Y$ (in this paper energy consumption), $\bar{Y}$ is the mean of actual value, $\mathrm{n}$ is the number of data points.

The models with the best statistical values can be chosen from a list. If the calibrated models do not comply with the statistical values limit, the building data must be manually adjusted and the simulation and calibration process need to be repeated. Otherwise, the model is considered calibrated and can be used or as a baseline for the $\mathrm{M} \& \mathrm{~V}$ or for the application of different retrofitting scenarios.

\section{Pilot Description}

\section{Building Characterisation}

This study researched a one-storey residential dwelling located on the island of Inis Mór (largest of the Aran Islands), Co. Galway off the west coast of Ireland. The dwelling was constructed in 1998, with additional 
upgrades in 2008 and 2015. The building is north facing with a total floor area of $110 \mathrm{~m}^{2}$.

The layout is shown in Figure 3 and the room types include bedrooms, a kitchen, glazed offices, circulation space, toilets and living rooms.

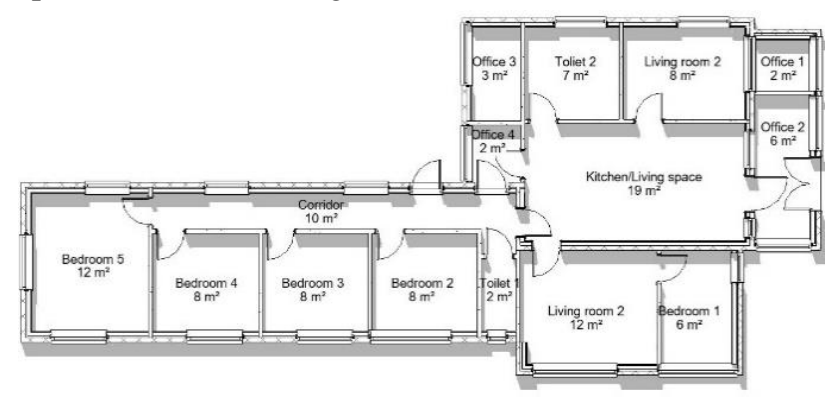

Figure 3-BIM model of the pilot building

Since 2012, the island of Inis Mór embarked on an ambitious program to reduce the dependence on fossil fuels and introduced decarbonisation of residential heating systems on the island with the ambition of becoming a carbon neutral island by 2025 (The Irish Government, 2009). Thus, the dwelling has a $5.5 \mathrm{~kW}$ heat pump, a variety of storage heaters and one $2 \mathrm{~kW}$ photovoltaic (PV) panel. Building data was collected through physical measurements, an interactive process of interviews and direct communication with the homeowner.

The building is currently one of the many dwellings that compries the Irish Pilot in the H2020 RESPOND Project (2017). RESPOND will deliver demand response solution from building residential level to district. The developed ROM will be used within the project to thermal forecasting and Measurament \&Verification baseline definition.

\section{Building Fabric}

The building consists of two types of external walls. The first, is a $4 \times 2 \mathrm{~m}$ timber frame, with $100 \mathrm{~mm}$ of wool glass insulation. The second, includes the addition of $50 \mathrm{~mm}$ fibre glass board insulation.

A weighted average of both walls was carried out to calculate the overall $\mathrm{U}$-value $(\mathrm{W} / \mathrm{m} 2 \mathrm{~K})$. The dwelling has a "warm roof" which offers optimal thermal performance and comprises of additional fibre glass board insulation on top of the existing roof structure. All windows and roof lights are timber framed and double glazed with $10 \%$ of the window openings face the east-west sides of the building and $40 \%$ face the north-south sides. The ground floor is a concrete slab with polystyrene insulation.

\section{Occupancy Schedules}

The dwelling houses a married couple with four children. Three children are studying away from home but return on holidays and at summer. Occupancy varies in this regard, but from months January to May, the house is fully occupied with 3 people in the morning, at least 1 person in the afternoon and fully occupied again in the evening.

\section{Heating Systems}

Air conditioning is non-existent within the dwelling, with the exception of extractor hoods used during cooking.

Temperature control within the dwelling focuses solely on heating rather than cooling, mostly between the months of September through to May, inclusive. The dwelling is equipped with a $5 \mathrm{~kW}$ air sourced Daikin heat pump which is currently only used for DHW purposes. Electrical storage heaters are located in the kitchen, bedrooms and living rooms. The storage heaters range from $1.3 \mathrm{~kW}$, $1.95 \mathrm{~kW}$ and $2.6 \mathrm{~kW}$ depending on room dimensions. Heating is not in use in the glazed offices, circulation space or toilets.

\section{Energy Consumption Analysis}

Through a detailed investigation it was found that there are five common power consuming products in this dwelling. The clothes machine has a power usage of $500 \mathrm{~W}$ and is used for one hour most mornings. The dishwasher of $1200 \mathrm{~W}$ is used on average for one hour each evening. The fridge runs throughout the day with a power consumption of $300 \mathrm{~W}$. Tumble dryer $(1400 \mathrm{~W})$ is used in direct correlation to the weather and more frequently in summer months as it rains quite often. The lighting in each room are energy efficient LED bulbs.

A list of the main building characteristic is described in the Table 1.

\section{Weather data}

The Aran Islands average temperature ranges from $14^{\circ} \mathrm{C}$ in summer to $6^{\circ} \mathrm{C}$ in winter. The Island is exposed to the elements and the prevailing winds are West/South West.

To retrieve the latest energy data for the simulations, the MERRA 2 application (Gelaro et al., 2017) was utilised to extract temperature, relative humidly, pressure and solar radiation for 2016 and 2017.

\section{Utility Bills analysis}

A full review of all energy bills dating back to 2006 were retrieved from the electricity provider. In particular, the electricity usage and charges were studied for years 2017 and 2016 to generate a typical profile of electricity use and to also understand any anomalous months.

Table 1 - Summary description table of pilot building

\begin{tabular}{|c|c|c|}
\hline Location & Latitude:53.13349 & Longitude: -9.696486 \\
\hline Dimensions & Floor area: $131 \mathrm{~m}^{2}$ & Volume: $313 \mathrm{~m}^{3}$ \\
\hline \multirow{4}{*}{$\begin{array}{l}\text { Main } \\
\text { construction } \\
\text { layers }\end{array}$} & \multicolumn{2}{|c|}{$\begin{array}{l}\text { Exterior walls } 2008\left[\mathrm{U} \text {-value }=0.20 \mathrm{~W} / \mathrm{m}^{2} \mathrm{~K}\right]: \text { Thin plaster }(15 \mathrm{~mm}) \text {, air cavity }(15 \mathrm{~mm}) \text {, wool glass insulation } \\
(100 \mathrm{~mm}) \text {, board insulation }(50 \mathrm{~mm}) \text {, hardwood }(120 \mathrm{~mm}) ;\end{array}$} \\
\hline & \multicolumn{2}{|c|}{$\begin{array}{l}\text { Exterior walls } 1997\left[\mathrm{U} \text {-value }=0.27 \mathrm{~W} / \mathrm{m}^{2} \mathrm{~K}\right] \text { : Thin plaster }(15 \mathrm{~mm}) \text {, air cavity }(15 \mathrm{~mm}) \text {, wool glass } \\
\text { insulation }(100 \mathrm{~mm}), \text { Hardwood }(120 \mathrm{~mm}) ;\end{array}$} \\
\hline & \multicolumn{2}{|c|}{ Windows $\left[\mathrm{U}\right.$-value $\left.=3.3 \mathrm{~W} / \mathrm{m}^{2} \mathrm{~K}\right]$ : Double glazed/timber frame; } \\
\hline & \multicolumn{2}{|c|}{$\begin{array}{l}\text { Roof }\left[\mathrm{U}-\text { value }=0.12 \mathrm{~W} / \mathrm{m}^{2} \mathrm{~K}\right] \text { : Tougher felt metal }(10 \mathrm{~mm}) \text {, board insulation }(100 \mathrm{~mm}) \text {, Tougher felt metal } \\
(10 \mathrm{~mm}) \text {, board insulation }(150 \mathrm{~mm}) \text {, thin plaster }(15 \mathrm{~mm}) .\end{array}$} \\
\hline
\end{tabular}




\section{Results and Discussion}

The exanimated workflow has been utilized against a detailed model, typically used in the IPMVP ${ }^{\circledR}$ Option D, and a whole building linear regression, typically used for the Option C, to demonstrate the accuracy of the ROM as $\mathrm{M} \& \mathrm{~V}$ baseline. Moreover, once the two simulation models have been calibrated, a comparison side by side has been done to demonstrate the capability of the ROM in simulating retrofitting scenarios.

The detailed model (Option D) is developed using the IES-VE 2017 software. The model is based on information collected during the data collection and investigation stage. Thermal templates of each room type were created to match the actual building. All simulations were performed using apacheSIM and each iteration was recorded in a manual calibration process.

Option $\mathrm{C}$ has been developed using a linear regression model based on monthly energy bills (12 months as recommended by IPMVP ${ }^{\circledR}$ ), occupancy schedules and HDD.

The ROM is utilised as outlined in the work-flow in the previous Methodology section. The parameters collected are inputted into the Tool and each parameter estimation was inserted in ROMFit. In the following Table 2, the list of resistances and capacitance calculated by the Tool has been provided.

Table 2 - Aran Island ROM resistances and capacitances

\begin{tabular}{|c|c|c|c|c|c|}
\hline & Value & Unit & & Value & Unit \\
\hline RWALL_IS & 5.09E-04 & $\mathrm{K} / \mathrm{W}$ & $\mathbf{R}_{\text {M_IS }}$ & 1.07E-03 & $\mathrm{K} / \mathrm{W}$ \\
\hline Rwall & 8.29E-03 & $\mathrm{K} / \mathrm{W}$ & $\mathbf{R}_{\mathbf{M}}$ & $5.44 \mathrm{E}-03$ & $\mathrm{~K} / \mathrm{W}$ \\
\hline RWALL_ES & $1.56 \mathrm{E}-04$ & $\mathrm{~K} / \mathrm{W}$ & $\mathrm{C}_{\mathrm{M}}$ & $4.19 \mathrm{E}+07$ & $\mathrm{~J} / \mathrm{K}$ \\
\hline Cwall & $2.32 \mathrm{E}+07$ & $\mathrm{~J} / \mathrm{K}$ & RGF_IS & 9.94E-04 & $\mathrm{K} / \mathrm{W}$ \\
\hline RwIN_IS & 2.83E-03 & $\mathrm{K} / \mathrm{W}$ & $\mathbf{R}_{\mathbf{G F}}$ & $1.55 \mathrm{E}-02$ & $\mathrm{~K} / \mathrm{W}$ \\
\hline RwIN & $6.60 \mathrm{E}-03$ & $\mathrm{~K} / \mathrm{W}$ & $\mathbf{R}_{\text {GF_ES }}$ & $3.06 \mathrm{E}-04$ & $\mathrm{~K} / \mathrm{W}$ \\
\hline$R_{\text {WIN }}$ & $6.60 \mathrm{E}-03$ & $\mathrm{~K} / \mathrm{W}$ & $\mathrm{C}_{\mathrm{GF}}$ & $3.60 \mathrm{E}+07$ & $\mathrm{~J} / \mathrm{K}$ \\
\hline Rwin_es & $8.72 \mathrm{E}-04$ & $\mathrm{~K} / \mathrm{W}$ & & & \\
\hline
\end{tabular}

Since the values of the previous table are calculated with the real specifications of the building construction, they are not considered in the calibration process because they have a low level of uncertainty. The values that are used in the calibration process are those with a high-level uncertainty. Table 3 outlines the model building parameters calculated by ROMfit for the calibration procedure.

Table 3 - Values of uncertain parameter calculated by ROMFit

\begin{tabular}{|c|c|c|}
\hline $\begin{array}{c}\text { Uncertain } \\
\text { parameter }\end{array}$ & Description & Value \\
\hline AlphaH & Heating - efficiency/utilisation & 0.1 \\
\hline AlphaC & Cooling - efficiency/utilisation & - \\
\hline L_rate & Air infiltration rate $[\mathrm{kg} / \mathrm{s}]$ & 2 \\
\hline AlphaEq & Equipment - efficiency/utilisation & 0.1 \\
\hline AlphaLig & Lighting - efficiency/utilisation & 0.5 \\
\hline
\end{tabular}

\section{Baseline period energy demonstration}

Once the three models have been developed and calibrated with 2017 data, a comparison in terms of accuracy as IPMVP ${ }^{\circledR}$ baseline period have been carried out. As it can be seen in Table 4, the ROM proves to be the most accurate statistically. With this, the ROM proved to be the least time consuming to calibrate in comparison to its IES-VE counterpart. This is mainly due to the sheer number of parameters involved in calibrating the whitebox IES-VE model.

Table 4-Calibrated models' statistical values, using 2017 data

\begin{tabular}{|c|c|c|c|}
\hline Model & ROM & IES-VE & Lin-REg \\
\hline Total energy & $12568 \mathrm{kWh}$ & $12527 \mathrm{~kW}$ & - \\
\hline NMBE & $-0.14 \%$ & $0.21 \%$ & - \\
\hline CV(RMSE) & $6.05 \%$ & $7.57 \%$ & $13.89 \%$ \\
\hline $\mathbf{R}^{\mathbf{2}}$ & 0.98 & 0.97 & 0.91 \\
\hline
\end{tabular}

As suggested by the IPMVP ${ }^{\circledR}$ methodology, a comparison was made of the three models, in terms of monthly deviation to measured data. From Table 5 , it is evident that both the ROM and the IES-VE model satisfy the criteria set out as they can keep the monthly deviation within the $15 \%$ recommended by IPMVP ${ }^{\circledR}$. However, the ROM proves to be the most accurate as its monthly deviation always lies within $10 \%$ of measured data.

Table 5-Calibrated models' monthly deviation, using 2017 data

\begin{tabular}{|c|c|c|c|}
\hline \multirow{2}{*}{$\begin{array}{c}\text { Reference } \\
\text { period }\end{array}$} & \multicolumn{3}{|c|}{ Monthly deviation } \\
\cline { 2 - 4 } & ROM & IES-VE & LIN-REG \\
\hline $\mathbf{1 2}$ DEC - 9 JAN & $-3.1 \%$ & $13.0 \%$ & $-4.9 \%$ \\
\hline $\mathbf{9}$ JAN - 2 FEB & $-3.8 \%$ & $-10.8 \%$ & $2.3 \%$ \\
\hline $\mathbf{2}$ FEB - 13 MAR & $-5.2 \%$ & $-0.9 \%$ & $-0.2 \%$ \\
\hline $\mathbf{1 3}$ MAR - 7 APR & $8.5 \%$ & $-7.8 \%$ & $-12.0 \%$ \\
\hline $\mathbf{7}$ APR - 2 MAY & $-0.6 \%$ & $-0.1 \%$ & $21.5 \%$ \\
\hline $\mathbf{2}$ MAY - 19 JUN & $0.5 \%$ & $-6.6 \%$ & $7.1 \%$ \\
\hline 19 JUN - 12 JUL & $0.9 \%$ & $4.1 \%$ & $1.3 \%$ \\
\hline 12 JUL - 11 AUG & $-5.5 \%$ & $-4.4 \%$ & $20.2 \%$ \\
\hline 11 AUG -19 SEP & $-3.4 \%$ & $1.0 \%$ & $-4.0 \%$ \\
\hline 19 SEP - 11 OCT & $5.0 \%$ & $4.9 \%$ & $14.4 \%$ \\
\hline 11 OCT- 10 NOV & $9.8 \%$ & $-0.8 \%$ & $20.4 \%$ \\
\hline 10 NOV - 19 DEC & $-2.5 \%$ & $3.3 \%$ & $-0.2 \%$ \\
\hline
\end{tabular}

The ROM was then further validated by calibrating it with the previous 2016 year data. This data included weather file, occupancy schedules, setpoints, and heating ONOFF. The results obtained are similarly positive and satisfy all calibration criteria with a $\mathrm{NMBE}=0.62 \%, \mathrm{CV}$ $\mathrm{RMSE}=9.39 \%$. and $\mathrm{R}^{2}=0.976$.

In addition to this, Figure 4 outlines the monthly deviation using the 2016 data and shows that all months lie within the acquired $15 \%$ range. The months of January and December 2016 are not taken into consideration as the bills of these two months have been estimated by the energy provider. Therefore, we can assume that the ROM 
can be used for energy demand forecasting and $M \& V$ baseline definition.

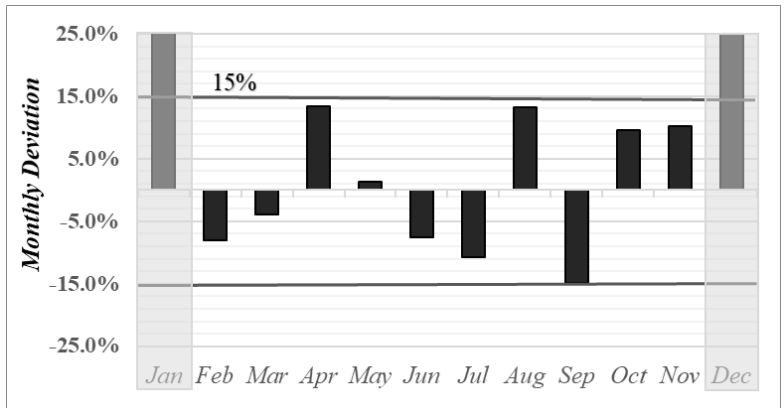

Figure 4 - ROM monthly deviation, validation using 2016 data

\section{Energy Cost Saving Measures (ECMs)}

In order to test the ROM in terms of renovation packages, close attention was paid to the back-casting method present in the IPMVP ${ }^{\circledR}$ 2016(Efficiency Valuation Organization, 2016). This method was utilised to model the building in its original conditions in the year 1998 . The original model could be then updated with each ECM implemented in the year 2008. Thus, the avoided energy consumption (AC) from each ECM was evaluated. During the renovation works in 2008 , the owner upgraded numerous parts of the house. This included an upgrade from single to double glazing windows (A), the addition of $50 \mathrm{~mm}$ of insulation in some parts of the old external wall (B) and an upgrade in insulation on the roof with the addition of a $150 \mathrm{~mm}$ rigid foam $(\mathrm{C})$.

Table 6 outlines the $\mathrm{U}$-values $\left(\mathrm{W} / \mathrm{m}^{2} \mathrm{~K}\right)$ before and after the ECMs.

Table 6-U-value before and after the ECMs

\begin{tabular}{|c|c|c|}
\hline & As it is $U$-value $\left[W / m^{2} K\right]$ & $1998 \mathrm{U}$-value $\left[\mathrm{W} / \mathrm{m}^{2} \mathrm{~K}\right]$ \\
\hline $\mathbf{A}$ & 3.3 & 5.8 \\
\hline B & 0.20 & 0.27 \\
\hline $\mathbf{C}$ & 0.12 & 0.29 \\
\hline
\end{tabular}

Firstly, both the reduced order and IES-VE model are simulated under 1998 conditions. Then, the renovation packages are applied one by one to each model. Thus, an estimation of the AC is calculated.

Table 7 - ROM and IES-VE ECMs energy savings comparison

\begin{tabular}{|c|c|c|c|c|c|}
\hline & \multicolumn{2}{|c|}{ ROM } & \multicolumn{2}{|c|}{ IES-VE } & \multirow{2}{*}{$\begin{array}{c}\mid \mathbf{A C}_{\text {ROM }} \\
-\mathbf{A C}_{\text {IES }} \mid \\
{[\%]}\end{array}$} \\
\hline & $\begin{array}{l}\text { Total } \\
{[\mathrm{kWh}]}\end{array}$ & $\begin{array}{l}\mathrm{AC}_{\mathrm{ROM}} \\
{[\%]}\end{array}$ & $\begin{array}{l}\text { Total } \\
{[\mathrm{kWh}]}\end{array}$ & $\begin{array}{l}\mathrm{AC}_{\mathrm{IES}} \\
{[\%]}\end{array}$ & \\
\hline 1998 & 15060 & - & 15496 & - & - \\
\hline $\mathbf{A}$ & 14196 & 5.74 & 14770 & 4.69 & 1.05 \\
\hline B & 14552 & 3.37 & 15191 & 1.97 & 1.40 \\
\hline $\mathbf{C}$ & 13527 & 11.24 & 14363 & 7.32 & 3.92 \\
\hline$A+B$ & 13680 & 9.16 & 14413 & 6.99 & 2.17 \\
\hline$A+C$ & 12667 & 15.89 & 13291 & 14.23 & 1.66 \\
\hline $\mathrm{B}+\mathrm{C}$ & 13259 & 11.96 & 13777 & 11.10 & 0.86 \\
\hline As it is & 12568 & 16.54 & 12527 & 19.16 & 2.62 \\
\hline
\end{tabular}

Table 7 outlines a comparison of the annual saving generated for each ECM and combined ECMs for both models. The last column represents the absolute value difference between the avoided energy consumption calculated with the ROM and with the IES model

The mean value of $\left|\mathrm{AC}_{\mathrm{ROM}^{-}} \mathrm{AC}_{\mathrm{IES}}\right|$ is $1.95 \%$ with a standard deviation of $1.06 \%$. Thus, we can assume that the ROM is suitable to estimate the energy savings of retrofitting scenarios.

\section{Conclusion}

This paper uses a novel methodology to support Measurement and Verification $(\mathrm{M} \& \mathrm{~V})$ by developing and utilising Reduced Order Model (ROM) to estimate the monthly energy consumption and supports accurate and reliable quantification of energy savings achieved through Energy Conservation Measures (ECMs).

Modelica was used to develop the ROM and that is based on a grey-box model proposed by (Giretti et al., 2018). An additional Tool has been created to estimate main parameters and simplify the ROM usage. The information needed to populate the tools has been gathered from available technical information, surveys and interviews. The remaining uncertain parameters are evaluated based on specific values, and then adjusted throughout an automatic calibration process (ROMFit). Indeed, starting from the Modelica ROM a Functional Mock-up Unit (FMU) has been generated using Jmodelica and a Python code to optimize the calibration and the uncertain parameters estimation has been created To demonstrate the accuracy of the methodology, the ROM proposed in the paper is compared with a whole facility linear regression (Option C IPMVP ${ }^{\circledR}$ ) and white box model developed with IES-VE (Option D IPMVP ${ }^{\circledR}$ ) for a typical historical one-storey residential dwelling located on the Island of Inis Mór, Co. Galway.

This paper concludes that the ROM is the most accurate between the three models when being utilized as IPMVP ${ }^{\circledR}$ baseline energy consumption. This model has a yearly $\mathrm{NMBE}=-0.14 \%$, a CV-RMSE $=6.05 \%$ and $\mathrm{a} \mathrm{R}^{2}=0.98$, compared to the energy 2017 monthly energy bills. Furthermore, a comparison in terms of annual saving generated by ECMs between the ROM and IES model was completed and shows the possibility to use the ROM in estimating the savings achieved through ECMs

Further research is needed to:

- Apply the methodology in other buildings and validate the methodology;

- Create a standalone or web interface to use the ROM and improve the calibration engine;

- Application on demand response scenarios and validation [RESPOND];

- Create and integrate a library, to take in to account the thermal comfort in the ROM.

\section{Acknowledgment}

This research work was funded by the Irish Research Council (IRC) - R2M Solution S.r.l Enterprise Partnership Scheme Postgraduate Scholarship 2017 under project ID EPSPG/2017/359 and the European Union under the RESPOND project with Grant agreement No. 768619. 


\section{References}

Air Force Civil Engineer Center ESPC Playbook. Available at: https://www.afcec.af.mil/Portals/17/documents/Ener gy/ESPCPlaybook11-08-2017.pdf?ver=2017-11-19171008-597

American Society of Heating, Refrigerating and AirConditioning Engineers. (2009). 2009 ASHRAE handbook: Fundamentals. Atlanta (USA).

Bacher, P. and Madsen, H. (2011). Identifying suitable models for the heat dynamics of buildings. Energy and Buildings, 43(7), 1511-1522.

Berthou, T. et al. (2014). Development and validation of a gray box model to predict thermal behavior of occupied office buildings. Energy and Buildings, 74, 91-100.

BSI British Standards (2003). Solar protection devices combined with glazing. Calculation of solar and light transmittance. Simplified method. (BS EN 13363-1)

Coakley, D., Raftery, P. and Keane, M. (2014). A review of methods to match building energy simulation models to measured data. Renewable and Sustainable Energy Reviews. Elsevier, 37, 123-141.

Edgars Augustins et al. (2018). Managing energy efficiency of buildings: analysis of ESCO experience in Latvia. Energy Procedia. Elsevier, 147, 614-623

Efficiency Valuation Organization (2016) 'Core Concepts, International Performance Measurement and Verification Protocol - EVO 10000-1'.

EnergyPlus. Available at: https://energyplus.net/

eQUEST. Available at: http://www.doe2.com/equest/

Foucquier, A. et al. (2013). State of the art in building modelling and energy performances prediction: A review. Renewable and Sustainable Energy Reviews, Elsevier 2013, 23, 272-288

Functional Mock-up Interface. Available at: https://fmistandard.org/downloads/

Fux, S. F. et al. (2014). EKF based self-adaptive thermal model for a passive house. Energy and Buildings, 68(PART C), 811-817.

Gelaro, R. et al. (2017) The Modern-Era Retrospective Analysis for Research and Applications, Version 2 (MERRA-2)'. Journal of Climate, 30(14), 54195454.

Ginestet, S. and Marchio, D. (2010). Retro and on-going commissioning tool applied to an existing building Operability and results of IPMVP'. Energy. Elsevier, 35(4), 1717-1723.

Giretti, A. et al. (2018). Reduced-order modeling for energy performance contracting. Energy and Buildings. Elsevier., 167, pp. 216-230.

Harb, H. et al. (2016) .Development and validation of grey-box models for forecasting the thermal response of occupied buildings. Energy and Buildings. Elsevier, 117, 199-207.

IBPSA Project 1. Available at: https://ibpsa.github.io/project1/

Integrated Environmental Solutions. Available at: https://www.iesve.com/

International Organization for Standardization (2017). Thermal performance of windows, doors and shutters -- Calculation of thermal transmittance -- Part 1: General (ISO 10077-1).

International Organization for Standardization (2017). Thermal performance of buildings -- Heat transfer via the ground -- Calculation methods (ISO 13370).

International Organization for Standardization (2007). Building components and building elements -Thermal resistance and thermal transmittance -Calculation method (ISO 6946).

JModelica.org. Available at: https://jmodelica.org/

Lauster, M. et al. (2014). Low order thermal network models for dynamic simulations of buildings on city district scale.Building and Environment, 73, 223-231.

Lee, P. et al. (2015). Risks in Energy Performance Contracting ( EPC ) projects. Energy \& Buildings. Elsevier , 92, 116-127.

Li, X. and Wen, J. (2014). Review of building energy modeling for control and operation. Renewable and Sustainable Energy Reviews, 37, 517-537

Müller, D. et al. (2016). AixLib - An Open-Source Modelica Library within the IEA-EBC Annex 60 Framework. Proceedings from BauSIM 2016, Dresden (Germany), 14-16 September 2016.

Perez, R. et al. (1990). Modeling daylight availability and irradiance components from direct and global irradiance. Solar Energy, 44(5), 271-289.

Remmen, P. et al. (2018). TEASER: an open tool for urban energy modelling of building stocks. Journal of Building Performance Simulation, Taylor and Francis Ltd, 84-98.

RESPOND Project. Available at: http://projectrespond.eu/

Reynders, G. et al. (2014). Quality of grey-box models and identified parameters as function of the accuracy of input and observation signals. Energy and Buildings, Elsevier, 82. 263-274.

Reynders, G. et al. 2015). Impact of the heat emission system on the identification of grey-box models for residential buildings. Energy Procedia. Elsevier, 78, 3300-3305.

The Irish Government (2009) 'National Renewable Energy Action Plan Ireland', 1-165.

Union, E. (2018) 'Directive (EU) 2018/844 of the European Parliament and of the Council of 30 May 2018 '. 